\title{
Volume-based growth tumor kinetics as a prognostic biomarker for patients with EGFR mutant lung adenocarcinoma undergoing EGFR tyrosine kinase inhibitor therapy: a case control study
}

\author{
Ji Hyun Lee ${ }^{1}$, Ho Yun Lee ${ }^{1 *}$, Myung-Ju Ahn², Keunchil Park², Jin Seok Ahn², Jong-Mu Sun² and Kyung Soo Lee ${ }^{1}$
}

\begin{abstract}
Background: We aim to determine whether volumetric assessment has the potential to serve as a prognostic biomarker, and to assess the relationship between longitudinal tumor data during treatment and prognosis in lung adenocarcinoma patients with sensitizing EGFR mutations treated with EGFR tyrosine kinase inhibitors (TKI).

Methods: We retrospectively assessed patients with EGFR-mutant stage IV lung adenocarcinoma who were treated with EGFR TKIs until disease progression. CT studies of 106 patients were quantitatively analyzed in terms of tumor size and volume by comparing baseline and follow-up CT scans obtained at every two treatment cycles. Tumor response was quantified using longitudinal measurements, and tumor growth kinetics was determined. Correlation with early surrogate parameters for tumor response evaluation such as change in size, volume, and response rate was performed. The Cox-proportional hazard model and Log-rank test were used to predict overall survival (OS).

Results: Responders based on the percent change in volume after four cycles of TKI therapy had a higher OS than non-responders $(P=0.035)$. The percent of volume and size changes after four cycles of TKI therapy were significantly correlated with TTP $(P<0.001)$.

Conclusion: Volume measurements and corresponding rates of growth appear to be helpful adjuncts for predicting survival in patients undergoing EGFR-TKI therapy.
\end{abstract}

Keywords: Tumor volume, Biomarkers, Prognosis, Growth kinetics, Quantitative analysis

\section{Background}

Lung cancer remains the leading cause of cancerassociated mortality worldwide for both men and women, and non-small cell lung cancer (NSCLC) is the most common form of lung cancer, accounting for approximately $80 \%$ of all cases [1]. Traditionally, many patients with NSCLC exhibit a poor prognosis because of the advanced stage of the tumor at the time of diagnosis. However, the introduction of epidermal growth

\footnotetext{
* Correspondence: hoyunlee96@gmail.com

'Department of Radiology, Samsung Medical Center, Sungkyunkwan

University School of Medicine, 81 Irwon-ro, Gangnam-gu, Seoul 135-710,

South Korea

Full list of author information is available at the end of the article
}

factor receptor (EGFR) tyrosine kinase inhibitors (TKI) for patients with advanced-stage lung adenocarcinoma harboring activating EGFR mutations has changed the clinical course and survival rates of the patients with this type of cancer dramatically [2-4].

Response Evaluation Criteria in Solid Tumors (RECIST)-based assessment, which is based on a single measurement of the largest tumor dimension, offers an easily understood approach to determine change in anatomic size during treatment as an indicator of tumor response [5]. Indeed, this approach is widely used because of its simplicity and good correlation with disease response and clinical outcomes [6]. However, such conventional criteria do not adequately account for 
tumors having a complex shape, morphology, or irregular boundary [7]. In addition, RECIST-based assessment does not take a multi-slice integrated understanding of tumor response into consideration.

Several authors have suggested that multi-slice assessment of the whole tumor can be used to overcome some of the difficulties associated with uni-dimensional measurement criteria [8-12]. Indeed, several reports involving volumetric assessment have demonstrated that it is more sensitive to tumor changes, more reliable and reproducible than linear measurements, and shows a stronger correlation with tissue biomarkers [9, 13-15]. However, to the best of our knowledge, there have been no reports comparing tumor response after TKI therapy between uni-dimensional and volumetric assessment in terms of prognostic factors in patients with adenocarcinoma containing an EGFR mutation. In addition, we hypothesized that serial measurements during treatment or early tumor response may provide a significant value for prognosis, therefore, the model for the tumor growth kinetics was implemented using the longitudinal volume measurements of lesions.

Thus, the goal of this study was to assess tumor response and survival in patients with sensitized EGFR mutation-positive adenocarcinoma patients treated with EGFR-TKIs using uni-dimensional and volumetric methods. Serial longitudinal measurements of tumor size and volume during the treatment were also evaluated to determine whether they might be useful as a prognostic factor in addition to early tumor response.

\section{Methods}

\section{Patients}

Our institutional review board approved this retrospective study (\# 2014-03-002) and informed consent was waived. We acquired our patient data from a clinical trial of EGFR TKIs for the treatment of NSCLC [16]. The study was a randomized phase II study for the effectiveness evaluation of gefitinib versus erlotinib in patients with advanced stage of NSCLC who had failed to show a positive response to previous chemotherapeutic agents and was conducted at Samsung Medical Center. Among them, patients with pathology-proven stage IV lung adenocarcinoma with activated EGFR mutations who received EGFR-TKI therapy between January 2007 and March 2011 as a second-line therapy were enrolled in this study. The patients were treated with the recommended doses of either erlotinib ( $150 \mathrm{mg} / \mathrm{d}$ orally) or gefitinib $(250 \mathrm{mg} / \mathrm{d}$ orally). Gefitinib or erlotinib was sequentially administered on days 2 to 16 . The treatment cycle was repeated every 3 weeks until the appearance of disease progression or the end of the study period was reached. Inclusion criteria were as follows: (a) patients who underwent TKI treatment that was repeated at three week intervals; (b) availability of baseline contrast- enhanced chest computed tomography (CT) performed prior to TKI therapy initiation; (c) and follow-up contrastenhanced CT performed after every two TKI cycles using the same imaging acquisition technique (Fig. 1). Exclusion criteria were as follows: (a) patients who stopped TKI treatment because of reasons other than progressive disease, including loss to follow-up, hopeless discharge, or drug side effect ( $\mathrm{n}=25)$; (b) patients who stopped receiving TKI therapy due to progressive disease in extra-thoracic organs without lung lesion progression $(n=13)$; (c) patients who failed to complete the first two cycles of TKI treatment because of progressive disease observed during the first follow-up $(n=6)$. (e) Patients who didn't have measurable primary lung lesion, who had diffuse hematolymphangitic metastasis, malignant effusion, or seeding lesions in the fissure or pleura $(n=83)$. As a result, a total of 106 patients $(\mathrm{M}: \mathrm{F}=41: 65$, median age at diagnosis, 58 years) were included in our study (Fig. 2). The median duration of TKI treatment was $11.9(2.2-34.6)$ months.

\section{CT examination and volume measurements}

All patients underwent contrast-enhanced helical CT with an eight- (LightSpeed Ultra, GE Healthcare) or 16detector row (LightSpeed 16, GE Healthcare) CT scanner during a breath-hold after injection of an iodinated contrast agent (100 mL of iopamidol: Iomeron 300; Bracco, Milan, Italy) at a rate of $1.5 \mathrm{~mL} / \mathrm{sec}$ using a power injector followed by a $20 \mathrm{cc}$ saline flush at a rate of $1.5 \mathrm{~mL} / \mathrm{sec}$. The thorax from the supraclavicular fossa to the renal hilum level was imaged 90 seconds after injection of the contrast agent with the following parameters: detector collimation, $0.625 \mathrm{~mm}$; field of view, $34.5 \mathrm{~cm}$; beam pitch, 1.35 or 1.375; gantry speed, $0.6 \mathrm{sec}-$ ond per rotation; $120 \mathrm{kVp} ; 150-200 \mathrm{~mA}$; and section thickness, $1.25 \mathrm{~mm}$ for transverse images. All imaging data were reconstructed using soft-tissue algorithms. CT scans were retrieved using the Picture Archiving and

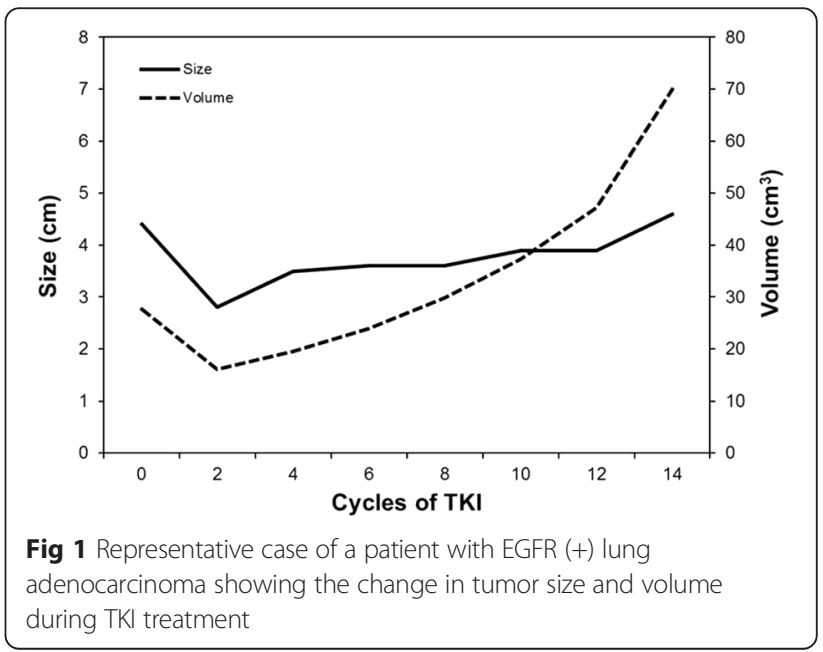




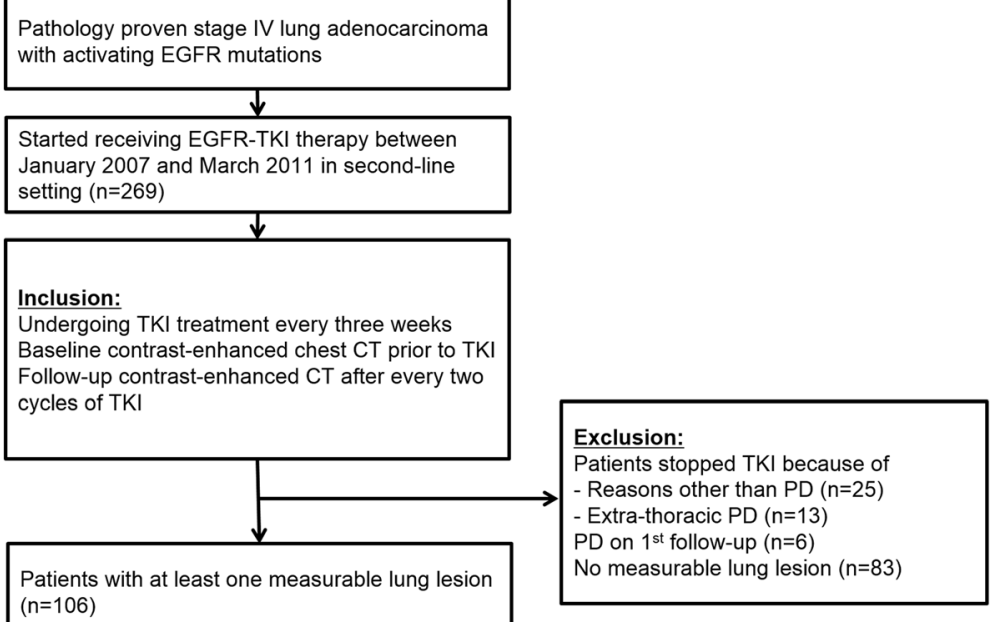

Fig 2 Flowchart for inclusion and exclusion of patients. TKl, tyrosine kinase inhibitors; PD, progressive disease

Communications System (PACS) (Centricity, GE Healthcare).

For each measureable lesion, the largest diameter was measured manually using the PACS tool. Volume measurements were also performed with a semi-automated method using MRIcro (version 1.40, Chris Rorden, University of Nottingham, Great Britain). The regions of interest (ROIs) were manually drawn for every slices in which the target lesions exist. The margins of the target lesions were drawn freehand (Fig. 3). The interval of the percent change in size and volume of lesions was calculated after two and four cycles (6 and 12 weeks, respectively) of TKI treatment, respectively.

\section{Parameter calculations}

Volume measurements of lesions were modeled using the tumor growth kinetics as shown in Fig. 4. $T_{0}, T_{r}$ and $\mathrm{T}_{\mathrm{p}}$ indicate the time of TKI initiation, the time when the lesion reached a nadir (the smallest tumor volume from baseline to TKI termination) based on volume measurement, and the time when TKI treatment was stopped due to disease progression. A more than $20 \%$ increase of the linear sums of the target lesions was defined as disease progression based on RECIST criteria [5]. $\mathrm{T}_{1}$ and $\mathrm{T}_{2}$ indicate the times of first and second follow up CT which were taken after two and four cycles of TKI, respectively. Likewise, $\mathrm{V}_{0}, \mathrm{~V}_{1}, \mathrm{~V}_{2}, \mathrm{~V}_{\mathrm{r}}$, and $\mathrm{V}_{\mathrm{p}}$ indicate the volume measurements at times $T_{0}, T_{1}, T_{2}, T_{r}$ and $T_{p}$, respectively. The time to nadir (TTN) and time to progression (TTP) were defined as the time intervals between $T_{0}$ and $T_{v}$ and between $T_{r}$ and $T_{p}$, respectively. The response rate $(\mathrm{RR})$ and progression rate $(\mathrm{PR})$ were calculated by dividing $\left(\mathrm{V}_{\mathrm{r}}-\mathrm{V}_{0}\right)$ by TTN and $\left(\mathrm{V}_{\mathrm{p}}-\mathrm{V}_{\mathrm{r}}\right)$ by TTP, respectively. $\Delta \mathrm{S} 1$ and $\Delta \mathrm{S} 2$ were used to indicate the percent change in tumor size based on unidimensional measurement at first and second follow up

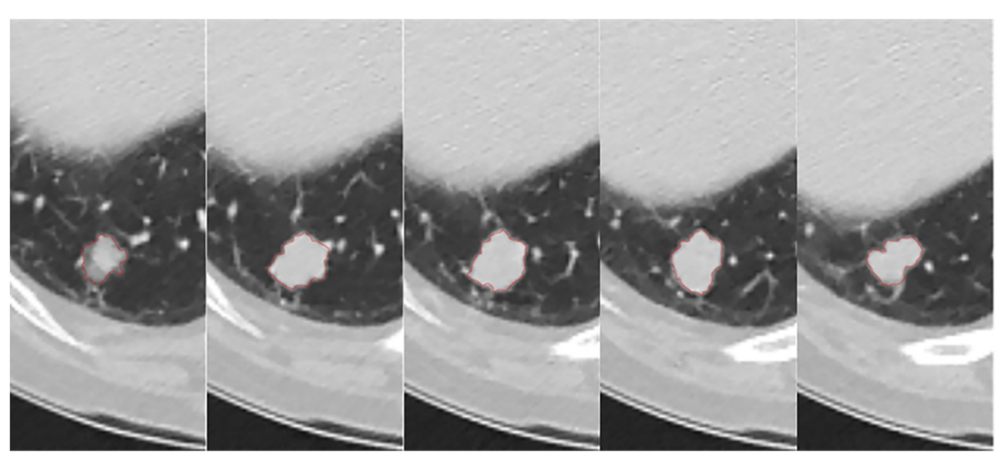

Fig $3 \mathrm{ROI}$ placement in five serial CT slices of a patient with EGFR (+) lung adenocarcinoma in the right lower lobe 


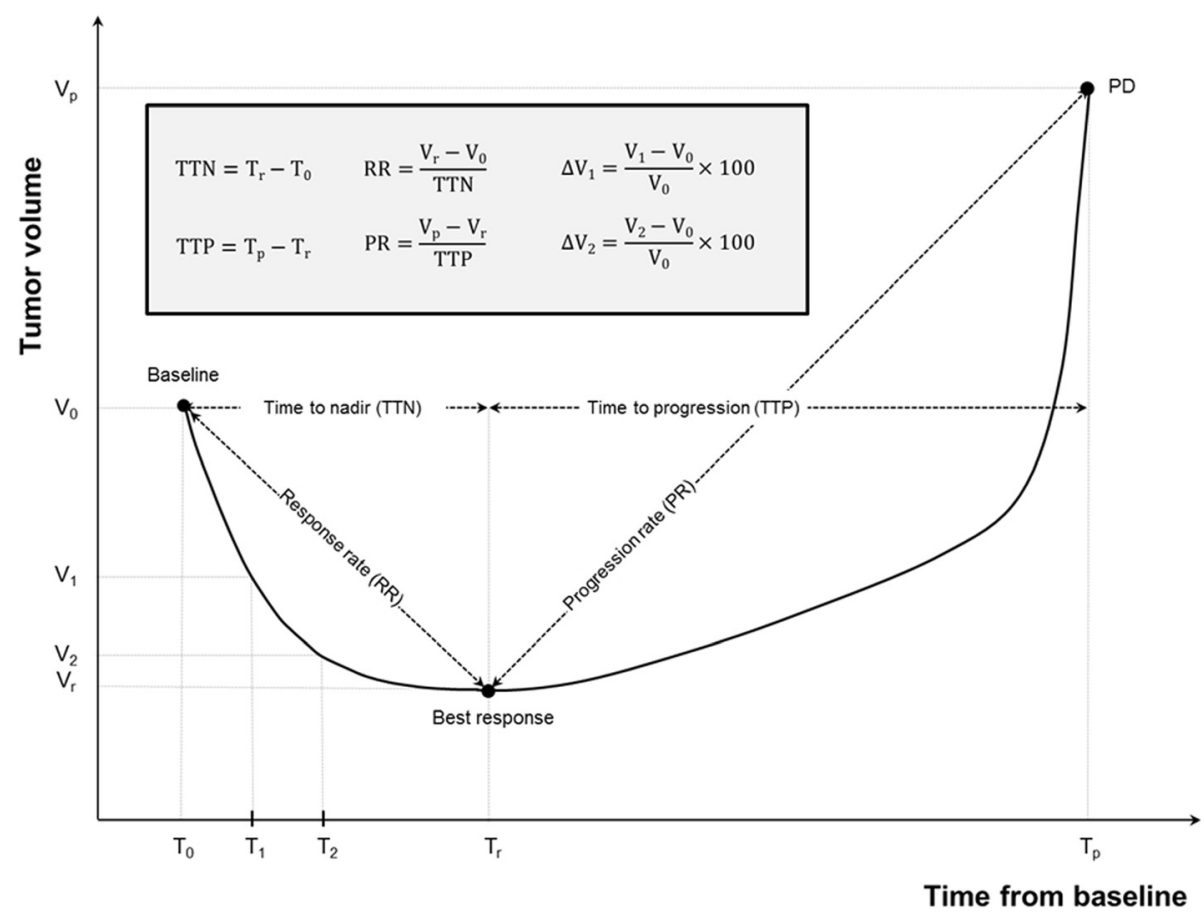

Fig 4 Conceptual graph showing tumor volume after TKI treatment. TKI, tyrosine kinase inhibitors; PD, progressive disease. $T_{0}$, the time of TKI initiation; $T_{1}$, the time of first follow up $C T$ after two cycles of $T K K_{;} T_{2}$, the time of second follow up CT after four cycles of TKl; $T_{p}$, the time when the lesion reached a nadir; $V_{n}$, the volume measurements at time $T_{n}(n=0,1,2, r, p) ; T_{1}, T_{2}, T_{r}$, and $T_{p}$, respectively $V_{1}$, the tumor volume at the time of $\mathrm{T}_{1} ; \mathrm{V}_{2}$, the tumor volume at the time of $\mathrm{T}_{2} ; T \mathrm{~N}$, time to nadir; TTP, time to progression; RR, response rate; PR, progression rate

which were performed after two and four cycles of TKI treatment, respectively, while $\Delta \mathrm{V} 1$ and $\Delta \mathrm{V} 2$ were used to define changes in tumor volume in the same manner.

\section{Statistical analysis}

Univariate and multivariate analyses were performed to assess covariate effects on overall survival (OS) using a Cox proportional hazard model with $95 \%$ confidence intervals. Survival curves were derived using KaplanMeier methods for OS and were compared using the log-rank test. Hazard ratios were calculated by adopting the Cox proportional hazard model. The association between time to progression (TTP) and early surrogate parameters for tumor response evaluation, which involved the variables $\Delta \mathrm{S} 1, \Delta \mathrm{S} 2, \Delta \mathrm{V} 1, \Delta \mathrm{V} 2, \mathrm{RR}$, and TTN, were calculated by Pearson correlation. Statistical analysis was performed using SPSS version 18 statistical software for Windows (SPSS; SPSS Inc; Chicago, IL). $P$ values less than or equal to 0.05 were considered statistically significant.

\section{Results}

\section{Patients}

Patient characteristics are summarized in Table 1. Among 106 patients, 87 had a single measureable lung lesion. A total of ten patients had two measurable lesions, while nine patients had three or more. Fifty-eight patients had an exon 19 microdeletion, 42 had the L858R mutation, and six had other mutations including insertion and missense mutations. The mean duration of TKI treatment was 352 days $(67$ - 1037). Thirty-five patients died during the follow-up period.

\section{Prognostic parameters for overall survival (OS)}

The radiologic parameters associated with reduced OS on univariate analysis were $\Delta \mathrm{V} 2$ (hazard ratio [HR] 0.980; $95 \% \mathrm{CI}, 0.965-0.995 ; P=0.011$ ), TTP (HR 1.024; $95 \% \mathrm{CI}, 1.009-1.039 ; P=0.001$ ), and RR (HR $0.500 ; 95 \% \mathrm{CI}, 0.263-0.950 ; P=0.034)$. Multivariate analysis corroborated these results, indicating that $\Delta \mathrm{S} 2$ (HR 0.981; 95 \% CI, $0.965-0.998 ; \mathrm{P}=0.026), \Delta \mathrm{V} 2$ (HR $0.976 ; 95 \% \mathrm{CI}, 0.957-0.995 ; P=0.012$ ), TTP (HR 3.155; $95 \% \mathrm{CI}, 2.809-3.544 ; P<0.001$ ), and RR (HR 0.597; $95 \% \mathrm{CI}, 0.378-0.941 ; P=0.026)$ were independent and significant predictors of OS (Table 2).

The median OS was 19.9 months $(3.1-72.5$ months, mean 20.5 months, 95 \% CI, 18.5 - 22.6). The mean duration of TKI treatment was 12.3 months $(2.2-34.5$ months; $95 \% \mathrm{CI}, 11.0$ - 13.8). We determined the association between patient subgroups based on $\Delta S 2, \Delta V 2$, RR, TTP and OS using the Kaplan-Meier method and log-rank test. Patients with a slow response rate (RR) (21.8 vs 48.5 months; cut-off, $\left.-7.5 \mathrm{~cm}^{3} / \mathrm{mo} ; P=0.026\right)$ or patients showing small volume reduction $(\Delta \mathrm{V} 2)$ (31.7 vs 
Table 1 Patient characteristics

\begin{tabular}{|c|c|}
\hline Age at diagnosis, years & 58 (range, 24-83) \\
\hline Total number of included lesions & 141 \\
\hline \multicolumn{2}{|l|}{ Gender } \\
\hline Male & $41(38.7)$ \\
\hline Female & $65(61.3)$ \\
\hline \multicolumn{2}{|l|}{ Smoking history } \\
\hline Never & $61(57.5)$ \\
\hline Former or current & $32(30.2)$ \\
\hline Unknown & $13(12.3)$ \\
\hline \multicolumn{2}{|l|}{ Stage at initial diagnosis } \\
\hline IV & $106(100)$ \\
\hline \multicolumn{2}{|c|}{ Number of included lesions in a patient } \\
\hline 1 & $87(82)$ \\
\hline 2 & $10(9)$ \\
\hline$\geq 3$ & $9(8)$ \\
\hline \multicolumn{2}{|c|}{ Site of included metastatic lesions (total 35) } \\
\hline Lung & $16(46)$ \\
\hline Liver & $9(17)$ \\
\hline Lymph node & $10(29)$ \\
\hline \multicolumn{2}{|l|}{ TKI drug } \\
\hline Gefitinib (Iressa $\left.{ }^{\oplus}\right)$ & $60(57)$ \\
\hline Erlotinib (Tarceva $\left.{ }^{\oplus}\right)$ & $46(43)$ \\
\hline \multicolumn{2}{|l|}{ EGFR Mutation subtype } \\
\hline Exon 19 deletion & $58(55)$ \\
\hline Exon 21 L858R & $42(40)$ \\
\hline Others & $6(6)$ \\
\hline
\end{tabular}

EGFR; epidermal growth factor receptor

TKl; tyrosine kinase inhibitors

Note-Unless otherwise indicated, data in parentheses are percentages

Table 2 Univariate and multivariate analyses for overall survival (OS)

\begin{tabular}{|c|c|c|c|c|}
\hline \multirow[b]{2}{*}{ Parameters } & \multicolumn{2}{|l|}{ Univariate } & \multicolumn{2}{|l|}{ Multivariate } \\
\hline & Hazard ratio & $P$ values & Hazard ratio & $P$ values \\
\hline$\Delta \mathrm{S} 1$ & $1.012(0.983,1.042)$ & 0.422 & $1.015(0.985,1.046)$ & 0.329 \\
\hline$\Delta \mathrm{S} 2$ & $0.983(0.963,1.004)$ & 0.119 & $0.981(0.965,0.998)$ & 0.026 \\
\hline$\Delta \mathrm{V} 1$ & $0.989(0.971,1.007)$ & 0.234 & $0.995(0.973,1.017)$ & 0.648 \\
\hline$\Delta \mathrm{V}_{2}$ & $0.980(0.965,0.995)$ & 0.011 & $0.976(0.957,0.995)$ & 0.012 \\
\hline$\pi \mathrm{T}$ & $0.994(0.986,1.003)$ & 0.176 & $0.997(0.985,1.008)$ & 0.574 \\
\hline TTP & $1.024(1.009,1.039)$ & 0.001 & $3.155(2.809,3.544)$ & $<0.001$ \\
\hline $\mathrm{RR}$ & $0.500(0.263,0.950)$ & 0.034 & $0.597(0.378,0.941)$ & 0.026 \\
\hline$P R$ & $1.056(0.857,1.301)$ & 0.608 & $1.042(0.855,1.270)$ & 0.684 \\
\hline
\end{tabular}

$\Delta \mathrm{S} 1$, the percent change in tumor size based on uni-dimensional measurement at the first follow up; $\Delta \mathrm{S} 2$, the percent change in tumor size based on uni-dimensional measurement at the second follow up; $\Delta \mathrm{V} 1$, the percent change in tumor volume at the first follow up; $\Delta \mathrm{V} 2$, the percent change in tumor volume at the second follow up, $T T N$; time to nadir, $T T P$; time to progression; RR, response rate, $P R$; progression rate

Unless otherwise indicated, data in parentheses are $95 \%$ confidence intervals
43.3 months; cut-off, $-35 \%$; $P=0.035$ ) were more highly associated with a poor prognosis. In addition, a short time to progression (TTP) significantly decreased OS (21.6 vs 50.6 months; cut-off, 7.7 months; $P<0.001)$. The correlations of OS with $\Delta \mathrm{S} 2$ (40.3 vs 41.3 months; cut-off, $-20 \%$, $P=0.956$ ) was not significant (Fig. 5).

\section{Association between TTP and early surrogate parameters for tumor response evaluation}

Having determined that TTP exhibited the strongest correlation with OS among several response parameters, we next assessed the correlation between TTP and early surrogate parameters including $\Delta S 1, \Delta S 2, \Delta V 1, \Delta V 2, R R$ and TTN. $\Delta S 2$ (correlation coefficient, $-0.254 ; P<0.001$ ) and $\Delta$ V2 (correlation coefficient, $-0.277 ; P<0.001$ ) were significantly associated with TTP by Pearson correlation (Table 3).

\section{Discussion}

There are many strong supporters of RECIST because it is an easily understood method that allows simple ruler analysis of printed films as well as workstation use of electronic calipers to produce results readily calculated on scratch paper. However, RECIST does not utilize multi-slice integrated data. In addition, the evaluation of tumor response is very complicated when the tumor has a complex shape and irregular or diffuse boundaries. Furthermore, a possible dichotomy between "objective tumor response" and "clinical improvement" has been suggested [17]. In two large phase III trials for patients with metastatic colorectal cancer, response rates based on RECIST and WHO criteria were found to poorly reflect patient benefit compared with progression-free survival and percentage of patients experiencing tumor control [18].

As an alternative to linear measurement-based RECIST, volumetric assessment is a more sensitive technique capable of identifying changes in tumor size $[8,12]$. Specifically, volumetric assessment reflects morphologic changes of tumors, including those that show no change in longest diameter. In addition, volumetry is more reliable and reproducible, has excellent inter- and intra-observer agreement, and eliminates variability from reader decisions, all of which indicate that this approach may be useful in clinical practice [9-12]. In addition, recent studies regarding volumetric assessment have suggested that volumetric assessment may be used as a prognostic tumor biomarker [13] to determine patient prognosis [19]. Notably, Prasad et al. [20] reported that volumetric measurement yields different results for treatment response in a considerable percentage of patients with liver metastases originating from breast cancer compared with that of uni-dimensional or bi-dimensional assessment. Despite the fact that volumetric measurements require a 

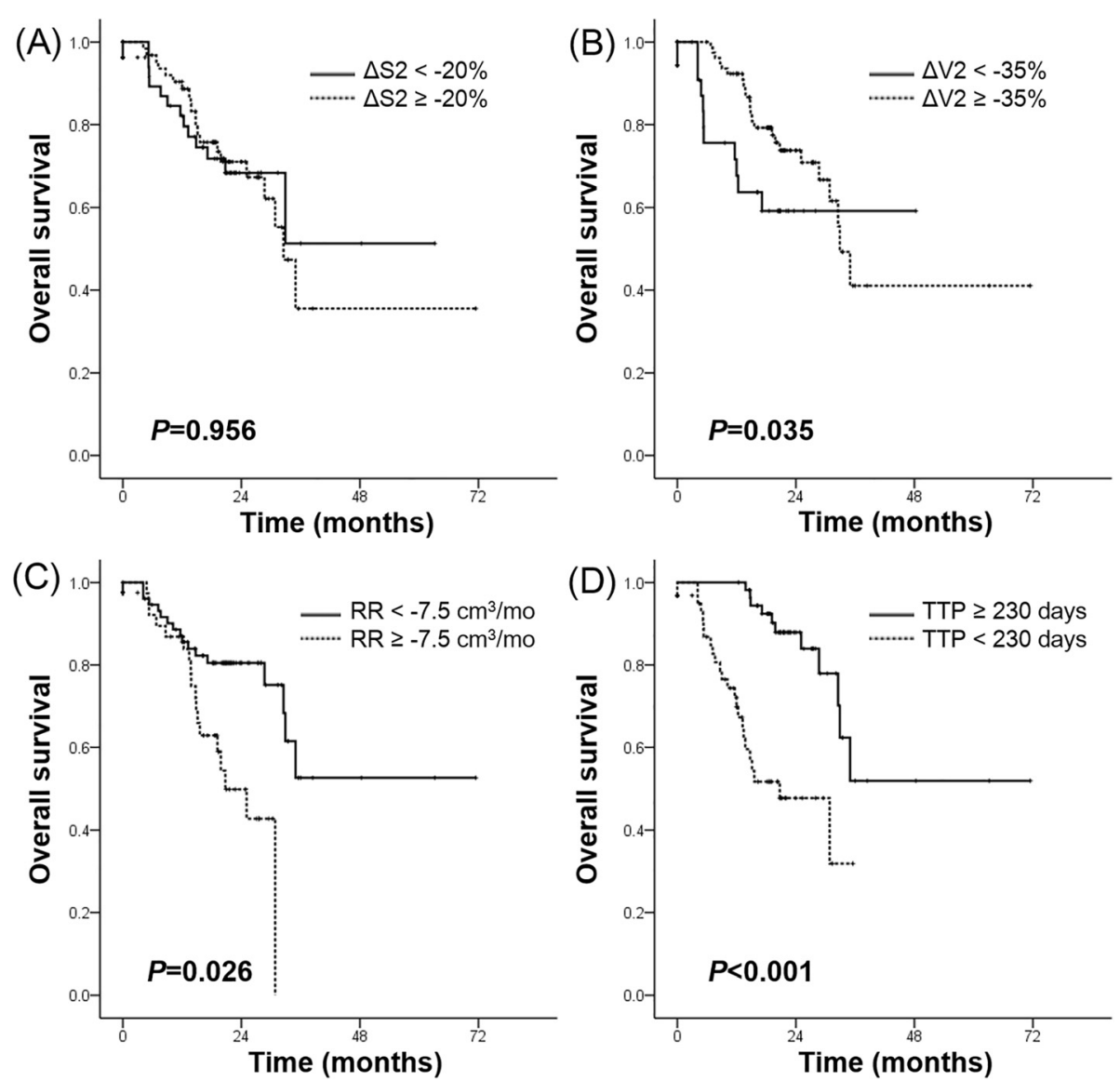

Fig 5 Kaplan-Meier survival curves for overall survival according to (a) size and (b) volume change after four cycles of TKI treatment, (c) response rate, and (d) time to progression. $\Delta \mathrm{S} 2$, the percent change in tumor size based on uni-dimensional measurement at the second follow up; $\Delta \mathrm{V} 2$, the percent change in tumor volume at the second follow up; TTP, time to progression; RR, response rate. TKI, tyrosine kinase inhibitors

substantial amount of time and effort, this approach is nonetheless expected to improve decision making in the treatment of cancer due to its reproducibility and greater sensitivity for the diagnosis of disease progression $[9,14,15]$. Recently, the introduction of semi-

Table 3 Correlations between early surrogate parameters and TाP

\begin{tabular}{lll}
\hline & Correlation coefficient & $P$ values \\
\hline$\Delta \mathrm{S} 1$ & -0.122 & 0.152 \\
$\Delta \mathrm{S} 2$ & -0.254 & $<0.001$ \\
$\Delta \mathrm{V} 1$ & -0.226 & 0.154 \\
$\Delta \mathrm{V} 2$ & -0.277 & $<0.001$ \\
$\mathrm{RR}$ & -0.046 & 0.591 \\
TTN & 0.012 & 0.890 \\
\hline
\end{tabular}

$\Delta \mathrm{S} 1$, the percent change in tumor size based on uni-dimensional measurement at the first follow up; $\Delta S 2$, the percent change in tumor size based on uni-dimensional measurement at the second follow up; $\Delta \mathrm{V} 1$, the percent change in tumor volume at the first follow up; $\Delta \mathrm{V} 2$, the percent change in tumor volume at the second follow up, $T T N$; time to nadir, $\Pi T P$; time to progression, $R R$; response rate automated or automated contour techniques using various software programs has made volumetric measurement easier and less time-consuming, which makes volumetric analysis feasible in a clinical setting.

To the best of our knowledge, there have been no studies focusing on treatment response evaluated with CT characteristics including both uni-dimensional and volumetric measurement after TKI therapy as a prognostic factor in patients with lung adenocarcinoma harboring EGFR mutations. In the present study, we measured the volume of the whole tumor as well as the longest unidimensional diameter of the tumor. In addition, measurable metastatic lesions that were included in the chest CT were also evaluated. The percentiles for both size and volume change after four cycles of TKI treatment were significantly associated with OS. Importantly, the stronger prognostic value of the change in volume compared with that of size, as shown in Fig. 1, was consistent with published data $[8,9,11,19]$.

Response assessment for cancer treatment is traditionally dependent on comparison with tumor size from a previous 
evaluation, and thus cannot reflect a long-term tendency of tumor change during follow-up. We hypothesized that not only early surrogate parameters on short-term follow-up, but also continuous longitudinal data with a concept of growth kinetics may more accurately reflect tumor response and patient benefit. To this end, we employed TTN and TTP as time parameters and RR and PR as response parameters using longitudinal volume measurements, and subsequently assessed the association between these longitudinal data and OS. As a result, TTP and RR were also independent significant predictors for OS. Although early size change also showed potential as an independent factor associated with OS in the multivariate analysis, only small $\Delta \mathrm{V} 2$ (less volume reduction), delayed RR (slow response) and short TTP (rapid progression) were associated with poor prognosis. Claret et al. [21] also reported strong association between time to tumor growth (TTG) as a longitudinal tumor data and OS in patients with metastatic colorectal cancer. Compared with TTG, which was calculated based on the model predicted time to tumor size nadir, our TTN was calculated based on tumor volume measurement, because growth kinetics were derived from volume rather than size. On the other hand, our results showed no significant association between TTN and OS, contradicting the results of a previous study by Claret et al., and instead indicated that RR may be a potential prognostic factor. In addition, we determined that TTP, which refers to the time interval between tumor nadir based on volumetric measurement and time of PD, was the best metric to predict OS among early tumor change and longitudinal data. Several previous studies [22-25] reported that progression free survival (PFS) is a poor surrogate for OS for several tumors types including NSCLC, and thus longitudinal tumor data such as RR and TTP may be useful as a prognostic factor for OS. In addition, TTG identified by Claret et al. allows differentiation of drug effect from a favorable prognosis [26].

Our study has several limitations. First, our study was performed retrospectively at a single institution. To overcome this limitation, we attempted to enroll a large number of patients. Second, although we used a semiautomated method to measure tumor size and volume, there was a possibility of measurement error. Especially when the tumor was surrounded by an atelectatic lung or positioned close to the hila or pleura, differentiation between the tumor and adjacent normal anatomic structures was often difficult. In addition, despite using the semi-automated software, contouring irregular lesions was more time-consuming compared with uni-dimensional measurements. Also, the presence of different software and algorithms with no standard method can be another limitation of volumetric analysis. In spite of these disadvantages, further improvement in software is expected, which should minimize the drawbacks described above. Furthermore, objectivity and reproducibility, which are important issues concerning response parameters, could not be evaluated for volumetric assessment because independent measurement by different radiologists and repeated measurements by the same radiologists were not performed. Lastly, internal heterogeneity of tumors was not considered. Given the increasing understanding of molecular mechanisms of NSCLC in response and resistance to EGFR-TKI, additional radiographic strategies for objective response assessment and determination of progression are needed to better guide therapeutic decisions in EGFR-mutant NSCLC patients.

\section{Conclusion}

In conclusion, early radiologic parameters of tumors including size and volume change after TKI treatment can be used to predict treatment response and OS in EGFR mutant lung adenocarcinoma patients, with the stronger prognostic value of the change in volume compared with that of size. In addition, changing speed or response rate during serial treatment such as TTP and RR also showed potential as a prognostic factor. Therefore, volume measurements and evaluation of their changing speed appear to be helpful adjuncts for predicting patient survival in patients who are undergoing EGFR-TKI therapy as a second-line chemotherapeutic agent. Further studies are required to support systematic guidelines using volumetric analysis for tumor response assessment after treatment.

\section{Abbreviations}

NSCLC: non-small cell lung cancer; CT: computed tomography;

EGFR: epidermal growth factor receptor; HR: hazard ratio; OS: overall survival; PFS: progression free survival; PR: progression rate; RECIST: response evaluation criteria in solid tumors; RR: response rate; TKI: tyrosine kinase inhibitors; TTG: time to tumor growth; TTN: time to nadir; TTP: time to progression

\section{Competing interests}

The authors declare that they have no competing interests.

\section{Authors' contributions}

All authors read and approved the final manuscript. Study concept and design: JHL, HYL, MJA. Acquisition of data: MJA, KP, JSA, JMS. Analysis and interpretation of data: JHL, HYL, MJA, JMS. Drafting of the manuscript: JHL, HYL. Critical revision of the manuscript for important intellectual content: MJA. Statistical analysis: HYL.

\section{Acknowledgements}

This study has received funding through grants from the Korean Foundation for Cancer Research (KFCR-CB-2011-02-02).

\section{Author details}

${ }^{1}$ Department of Radiology, Samsung Medical Center, Sungkyunkwan University School of Medicine, 81 Irwon-ro, Gangnam-gu, Seoul 135-710, South Korea. ${ }^{2}$ Division of Hemato-Oncology of the Department of Internal Medicine, Samsung Medical Center, Sungkyunkwan University School of Medicine, Seoul 135-710, South Korea.

Received: 14 January 2016 Accepted: 1 March 2016

Published online: 16 March 2016 


\section{References}

1. Jemal A, Murray T, Ward E, Samuels A, Tiwari RC, Ghafoor A, et al. Cancer statistics, 2005. CA Cancer J Clin. 2005;55(1):10-30.

2. Takano T, Fukui T, Ohe Y, Tsuta K, Yamamoto S, Nokihara H, et al. EGFR mutations predict survival benefit from gefitinib in patients with advanced lung adenocarcinoma: a historical comparison of patients treated before and after gefitinib approval in Japan. J Clin Oncol. 2008;26(34):5589-95. doi:10.1200/JCO.2008.16.7254

3. Mok TS, Wu YL, Thongprasert S, Yang CH, Chu DT, Saijo N, et al. Gefitinib or carboplatin-paclitaxel in pulmonary adenocarcinoma. N Engl J Med. 2009; 361(10):947-57. doi:10.1056/NEJMoa0810699.

4. van Zandwijk N, Mathy A, Boerrigter L, Ruijter $H$, Tielen I, de Jong D, et al. EGFR and KRAS mutations as criteria for treatment with tyrosine kinase inhibitors: retro- and prospective observations in non-small-cell lung cancer. Ann Oncol. 2007:18(1):99-103. doi:10.1093/annonc/mdl323.

5. Jaffe CC. Measures of response: RECIST, WHO, and new alternatives. J Clin Oncol. 2006:24(20):3245-51. doi:10.1200/JCO.2006.06.5599.

6. Buyse M, Thirion P, Carlson RW, Burzykowski T, Molenberghs G, Piedbois P. Relation between tumour response to first-line chemotherapy and survival in advanced colorectal cancer: a meta-analysis. Meta-Analysis Group in Cancer. Lancet. 2000;356(9227):373-8.

7. Lee HY, Hyun SH, Lee KS, Kim BT, Kim J, Shim YM, et al. Volume-based parameter of 18)F-FDG PET/CT in malignant pleural mesothelioma: prediction of therapeutic response and prognostic implications. Ann Surg Oncol. 2010;17(10):2787-94. doi:10.1245/s10434-010-1107-z.

8. Goldmacher GV, Conklin J. The use of tumour volumetrics to assess response to therapy in anti-cancer clinical trials. Br J Clin Pharmacol. 2012; 73:846-54. doi:10.1111/j.1365-2125.2012.04179.x

9. Mozley PD, Bendtsen C, Zhao B, Schwartz LH, Thorn M, Rong Y, et al. Measurement of tumor volumes improves RECIST-based response assessments in advanced lung cancer. Transl Oncol. 2012;5(1):19-25.

10. Mozley PD, Schwartz LH, Bendtsen C, Zhao B, Petrick N, Buckler AJ. Change in lung tumor volume as a biomarker of treatment response: a critical review of the evidence. Ann Oncol. 2010;21(9):1751-5. doi:10.1093/ annonc/mdq051.

11. Nishino M, Guo M, Jackman DM, DiPiro PJ, Yap JT, Ho TK, et al. CT tumor volume measurement in advanced non-small-cell lung cancer: Performance characteristics of an emerging clinical tool. Acad Radiol. 2011;18(1):54-62. doi:10.1016/j.acra.2010.08.021.

12. Zhao B, Schwartz LH, Moskowitz CS, Ginsberg MS, Rizvi NA, Kris MG. Lung cancer: computerized quantification of tumor response-initial results. Radiology. 2006;241(3):892-8. doi:10.1148/radiol.2413051887.

13. Zhao B, Oxnard GR, Moskowitz CS, Kris MG, Pao W, Guo P, et al. A pilot study of volume measurement as a method of tumor response evaluation to aid biomarker development. Clin Cancer Res. 2010;16(18):4647-53. doi:10. 1158/1078-0432.CCR-10-0125.

14. Schwartz LH, Curran S, Trocola R, Randazzo J, Ilson D, Kelsen D. Volumetric 3D CT analysis-an early predictor of response to therapy. J Clin Oncol. 2007;25 Suppl 18:4576.

15. Altorki N, Heymach J, Guarino M, Lee H, Felip E, Bauer TI. Phase II study of pazopanib (GW786034) given preoperatively in stage I-II non-small cell lung cancer (NSCLC): a proof-of-concept study. Ann Oncol. 2008;19 Suppl 8:124.

16. Kim ST, Uhm JE, Lee J, Sun JM, Sohn I, Kim SW, et al. Randomized phase I study of gefitinib versus erlotinib in patients with advanced non-small cell lung cancer who failed previous chemotherapy. Lung Cancer. 2012;75(1): 82-8. doi:10.1016/j.lungcan.2011.05.022.

17. Fleming TR, DeMets DL. Surrogate end points in clinical trials: are we being misled? Ann Intern Med. 1996;125(7):605-13.

18. Grothey A, Hedrick EE, Mass RD, Sarkar S, Suzuki S, Ramanathan RK, et al. Response-independent survival benefit in metastatic colorectal cancer: a comparative analysis of N9741 and AVF2107. J Clin Oncol. 2008;26(2):183-9. doi:10.1200/jco.2007.13.8099.

19. Kozak MM, Murphy JD, Schipper ML, Donington JS, Zhou L, Whyte RI, et al. Tumor volume as a potential imaging-based risk-stratification factor in trimodality therapy for locally advanced non-small cell lung cancer. J Thorac Oncol. 2011:6(5):920-6.

20. Prasad SR, Jhaveri KS, Saini S, Hahn PF, Halpern EF, Sumner JE. CT tumor measurement for therapeutic response assessment: comparison of unidimensional, bidimensional, and volumetric techniques initial observations. Radiology. 2002:225(2):416-9. doi:10.1148/radiol.2252011604.
21. Claret L, Gupta M, Han K, Joshi A, Sarapa N, He J, et al. Evaluation of TumorSize Response Metrics to Predict Overall Survival in Western and Chinese Patients With First-Line Metastatic Colorectal Cancer. J Clin Oncol. 2013;31: 2110-4. doi:10.1200/jco.2012.45.0973.

22. Burzykowski T, Buyse M, Piccart-Gebhart MJ, Sledge G, Carmichael J, Lück HJ, et al. Evaluation of tumor response, disease control, progression-free survival, and time to progression as potential surrogate end points in metastatic breast cancer. J Clin Oncol. 2008;26(12):1987-92.

23. Collette L, Burzykowski T, Carroll KJ, Newling D, Morris T, Schröder FH. Is prostate-specific antigen a valid surrogate end point for survival in hormonally treated patients with metastatic prostate cancer? Joint research of the European Organisation for Research and Treatment of Cancer, the Limburgs Universitair Centrum, and AstraZeneca Pharmaceuticals. J Clin Oncol. 2005;23(25):6139-48.

24. Sherrill B, Amonkar M, Wu Y, Hirst C, Stein S, Walker M, et al. Relationship between effects on time-to-disease progression and overall survival in studies of metastatic breast cancer. Br J Cancer. 2008;99(10):1572-8.

25. Soria J, Massard C, Le Chevalier T. Should progression-free survival be the primary measure of efficacy for advanced NSCLC therapy? Ann Oncol. 2010; 21(12):2324-32

26. Maitland ML, Schwartz LH, Ratain MJ. Time to tumor growth: a model end point and new metric system for oncology clinical trials. J Clin Oncol. 2013; 31:2070-2. doi:10.1200/jco.2013.49.3635

\section{Submit your next manuscript to BioMed Central and we will help you at every step:}

- We accept pre-submission inquiries

- Our selector tool helps you to find the most relevant journal

- We provide round the clock customer support

- Convenient online submission

- Thorough peer review

- Inclusion in PubMed and all major indexing services

- Maximum visibility for your research

Submit your manuscript at www.biomedcentral.com/submit
) Biomed Central 